\title{
Enriched Rehabilitative Training Promotes Improved Forelimb Motor Function and Enhanced Dendritic Growth after Focal Ischemic Injury
}

\author{
Jeff Biernaskie and Dale Corbett \\ Division of Basic Medical Sciences, Faculty of Medicine, Memorial University of Newfoundland, St. John's, \\ Newfoundland, Canada A1B 3V6
}

\begin{abstract}
Chronic impairment of forelimb and digit movement is a common problem after stroke that is resistant to therapy. Previous studies have demonstrated that enrichment improves behavioral outcome after focal ischemia; however, postischemic enrichment alone is not capable of enhancing fine digit and forelimb function. Therefore, we combined environmental enrichment with daily skilled-reach training to assess the effect of intensive task-specific rehabilitation on long-term functional outcome. Rats were subjected to either endothelin-1-induced focal ischemia or sham surgery and subsequently designated to enriched-rehabilitation or standard-housing treatment groups starting $15 \mathrm{~d}$ after ischemia. Functional assessment of the affected forelimb at 4 and 9 weeks after treatment revealed that ischemic plus enrichment (IE) animals had improved $\sim 30 \%$ on the staircase-reaching task and were indistinguishable from sham animals for both latency and foot faults in a beam-
\end{abstract}

Behavior and experience are capable of inducing "plastic" changes within the normal (Rosenzweig et al., 1962; Greenough et al., 1985; Kolb and Gibb, 1991) and injured (Jones and Schallert, 1992; Nudo et al., 1996) (for review, see Kolb et al., 1998) adult brain. For example, exposure to an enriched environment before or after focal ischemic injury resulted in improved behavioral outcome (Ohlsson and Johansson, 1995; Johansson and Ohlsson, 1996) on several sensorimotor tasks. Similarly, specific behavioral experience, such as motor-skill training after experimental brain injury (Nudo et al., 1996) or constraint-induced therapy after clinical stroke (Taub et al., 1993; Kunkel et al., 1999; Liepert et al., 2000), provides substantial functional benefit. This suggests that rehabilitative therapies may remodel neuronal circuitry within the surrounding tissue and that such reorganization contributes to "recovery" of motor function compromised by the injury. Although recent studies have begun to investigate the mechanisms underlying sensorimotor recovery, such processes, as well as their time course, remain poorly understood.

Exposure to enriched living conditions initiated before or after focal ischemic injury in rats (Grabowski et al., 1993) has failed to

\footnotetext{
Received Feb. 13, 2001; revised April 25, 2001; accepted Apr. 26, 2001.

This work was supported by the Medical Research Council of Canada, by the Heart and Stroke Foundation of New Brunswick (D.C.), and by the Rick Hansen Neurotrauma Initiative (studentship to J.B.). We are very grateful to Kathy McKay, Dr. Jennifer Wells, Sue Evans, Rosemarie Farrell, and Andrea Darby-King for helpful advice and technical assistance on this project.

Correspondence should be addressed to Jeff Biernaskie, 4th Floor Basic Medical Sciences, Health Sciences Centre, Memorial University of Newfoundland, St. John's, Newfoundland, Canada A1B 3V6. E-mail: g83jab@mun.ca.

Copyright (C) 2001 Society for Neuroscience $0270-6474 / 01 / 215272-09 \$ 15.00 / 0$
}

traversing task. In contrast, ischemic plus standard (IS) animals remained significantly impaired on both tasks. Interestingly, both ischemic groups (IE and IS) relied on the nonaffected forelimb during upright weight-bearing movements, a pattern that persisted for the duration of the experiment. Dendritic arborization of layer $\mathrm{V}$ pyramidal cells within the undamaged motor cortex was examined using a Golgi-Cox procedure. IE animals showed enhanced dendritic complexity and length compared with both IS and sham groups. These results suggest that enrichment combined with task-specific rehabilitative therapy is capable of augmenting intrinsic neuronal plasticity within noninjured, functionally connected brain regions, as well as promoting enhanced functional outcome.

Key words: enrichment; rehabilitation; functional recovery; forelimb; motor cortex; dendritic arborization; stroke ameliorate forelimb and digit impairments, necessary for skilled reaching and grasping. By combining both enriched living conditions and daily skilled-reach training, we intended to provide necessary situations in which use of the affected limb would be increased, thereby effectively stimulating the compromised sensorimotor system. In addition, enrichment has been shown to alter expression of several neurotrophic growth factors, such as nerve growth factor (NGF) (Dahlqvist et al., 1999) and basic fibroblast growth factor (bFGF) (Rowntree and Kolb, 1997), and therefore may enhance recovery when initiated in concert with rehabilitation.

By exposing animals to enriched living conditions and daily rehabilitation, designed to encourage (rather than force) skilled use of the impaired forelimb, we attempted to improve sensorimotor function of the impaired forelimb and digits, as well as to provide an effective model for investigation of the mechanisms underlying rehabilitation-induced functional improvement. Importantly, treatment was delayed until $15 \mathrm{~d}$ after injury (Johansson and Ohlsson, 1996) to establish the efficacy of such therapy if introduced at a more "clinically relevant" time point, as well as to exclude possible deleterious effects of early behavioral overuse on recovery (Kozlowski et al., 1996; Risedal et al., 1999).

Rehabilitation after small ischemic lesions promotes neuronal reorganization within adjacent cortical tissue and is associated with motor recovery (Nudo et al., 1996). Recent clinical studies also indicate an ipsilateral contribution to functional recovery (Cramer et al., 1997; Cuadrado et al., 1999; Kopp et al., 1999). In addition, animal studies demonstrate electrolytic lesion-induced neuronal plasticity within functionally connected regions of the 

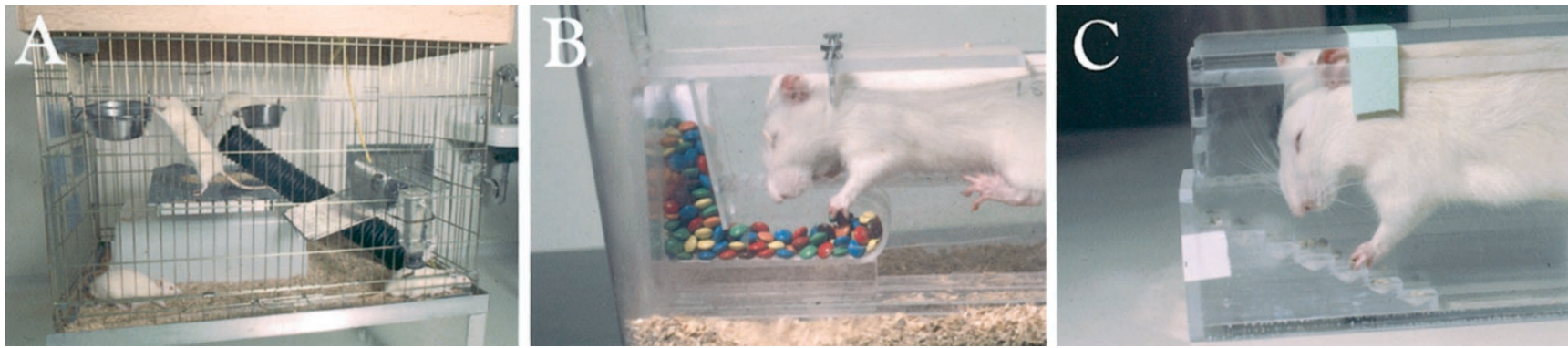

Figure 1. Enriched-rehabilitation treatment condition. A, View of a typical enriched environment. Cages and objects were changed twice weekly to promote exploration and a broad range of tactile experience. $B$, The rehabilitative reaching apparatus, which was implemented daily (5 d/week) to promote deficit-specific therapy of the impaired forelimb and digits. A shelf directly below the impaired limb was filled with food pellets to reinforce skilled use of the impaired forelimb. $C$, Side view of the staircase-reaching task. See Materials and Methods for additional description.

intact hemisphere (Jones and Schallert, 1994; Jones et al., 1999). The present experiment examined morphological plasticity within the intact hemisphere after ischemic injury that included both the sensorimotor cortex and striatum. Our objective was to assess the effect of enriched rehabilitation on dendritic reorganization within the intact hemisphere and to establish its potential importance to functional recovery. To investigate such neural substrates, a modified Golgi-Cox staining procedure was used to impregnate the dendritic arbor of layer $\mathrm{V}$ pyramidal neurons within the forelimb motor cortex of the undamaged hemisphere (ipsilateral to the impaired forelimb).

\section{MATERIALS AND METHODS}

\section{Subjects}

Subjects were 57 male Sprague Dawley rats (Charles River Laboratories, Montréal, Québec, Canada), each weighing 290-320 gm at the time of surgery. Animals were housed on a reverse $12 \mathrm{hr}$ light/dark cycle, and all behavioral assessments were done during the dark phase. All procedures were in accordance with guidelines established by the Canadian Council on Animal Care and received previous approval of the Institutional Animal Care Committee of Memorial University.

\section{Surgical procedures}

Anesthesia was induced using a mixture of $2 \%$ halothane in $30 \%$ oxygen and $70 \%$ nitrous oxide; animals were subsequently maintained using $1 \%$ halothane. Middle cerebral artery (MCA) occlusion was induced by stereotaxic microinjection of endothelin-1 (ET-1) (Sharkey et al., 1993; Sharkey and Butcher, 1995) to the distal portion of the MCA. Stereotaxic coordinates of the injection site with respect to bregma were as follows: anteroposterior, $+0.9 \mathrm{~mm}$; mediolateral, $-5.2 \mathrm{~mm}$; and dorsoventral, $-8.7 \mathrm{~mm}$. Endothelin-1 (60 pmol) was dissolved in $3 \mu \mathrm{l}$ of saline and injected over a period of $\sim 1 \mathrm{~min}$. The needle remained in place for $5 \mathrm{~min}$ to minimize backflow. Sham surgeries included either a $3 \mu$ linjection of saline adjacent to the MCA or all manipulations up to and including drilling the burr hole. Rectal temperature was monitored and maintained between 36.5 and $37.5^{\circ} \mathrm{C}$ using a heated water blanket for the duration of the surgery. In addition, body temperature was monitored for $8 \mathrm{hr}$ after surgery via intraperitoneal telemetry probes (Mini-Mitter Co., Sunriver, OR).

\section{Enriched rehabilitation}

Fifteen days after induction of focal ischemia, animals were designated to one of four groups: ischemia plus enrichment (IE), ischemia plus standard housing (IS), sham plus enrichment (SE), or sham plus standard housing (SS). "Enriched" animals were housed socially (groups of four to five animals) in large wire mesh cages (Fig. 1). Enrichment cages contained several different objects for exploration (i.e., shelves, plastic tubing, ladders, and rope) and were changed twice weekly; objects were rearranged after each cleaning. Animals had access to food and water ad libitum, except during behavioral testing.

In addition to being housed in enriched environments, enriched groups were exposed to daily rehabilitative training ( $5 \mathrm{~d} /$ week). Animals were removed from the enrichment cages and placed into standard rodent cages, each containing a Plexiglas rehabilitation reaching appa- ratus comprised of a central platform with a shelf on either side (Fig. 1, middle). The dimensions of the apparatus only allow independent forelimb retrieval of pellets on either side of the animal. The shelf below the unaffected forelimb was left empty, whereas the shelf below the impaired forelimb was filled with mini M\&Ms (which rats find highly palatable). The height to which M\&Ms were filled and the design of the apparatus prevented tongue use, or "cheating" with the unaffected forelimb. Animals had access to M\&Ms ad libitum for $6 \mathrm{hr}$ daily.

Standard groups were housed singly in Plexiglas cages and did not receive rehabilitative therapy. However, the standard condition was not completely impoverished. The repeated exposure to the staircase (two trials per day for $5 \mathrm{~d}$ ) and beam-walking test periods early in the recovery period (10-15 d after ischemia), as well as at later time points, provided novel behavioral experience directed toward limb use. To control for any possible effects of chocolate, sugar, or other additives on recovery, standard-treated animals were fed the average daily amount of M\&Ms eaten by an enriched animal (i.e., $\sim 9 \mathrm{gm} / \mathrm{d}$ ).

\section{Behavioral testing}

Staircase skilled-reaching test. Beginning the day before and continuing for the duration of the staircase testing period, animals were food restricted to $12 \mathrm{gm} / \mathrm{d}$. Body weights were recorded daily and did not fall below $80 \%$ of initial weight. Two weeks before surgery, animals were trained $(8 \mathrm{~d}, 2-15 \mathrm{~min}$ trials $/ \mathrm{d})$ to reach for pellets in the staircase test (Montoya et al., 1991). Each staircase consisted of seven steps, with three food pellets situated on each step. Animals were required to climb on a central platform to successfully retrieve and eat pellets $(45 \mathrm{mg}$; Noyes, Lancaster, $\mathrm{NH}$ ) from staircases situated on either side of the animal. The number of pellets eaten per side was used as a measure of forelimb reaching ability. The design of the apparatus prevented any dropped pellets from being retrieved. After surgery, animals were again tested for reaching ability at days $10-15$ (before treatment). That is, rats were tested for staircase deficits before being assigned to enriched rehabilitation or standard housing. They were retested at 4 (Post 1) and 9 (Post 2) weeks after initiation of enrichment or standard housing. Each test period consisted of 10 trials (two trials per day for $5 \mathrm{~d}$ ), and the best three trials were averaged to obtain the maximum level of performance. Animals that did not show a significant staircase deficit $(<65 \%$ of training performance) during the pretreatment test period (days 10-15 after surgery) were excluded from the experiment. To control for selection bias (before group designation), ischemic animals with similar reaching deficits were paired and then randomly assigned to either treatment condition.

Asymmetrical forelimb use. Animals were placed into a clear Plexiglas cylinder $(40 \mathrm{~cm}$ in diameter) situated on a glass tabletop and were videotaped from below, via an angled mirror. The number of forelimb wall contacts (single and bilateral) used for postural support was counted. The percentage of ipsilateral forelimb use was then calculated by using the following equation: (ipsilateral limb use/total number of limb uses) $\times$ 100 (Jones and Schallert, 1994; Forgie et al., 1996; Prusky and Whishaw, 1996).

Beam-traversing task. Before surgery, animals were trained to rapidly cross an elevated wooden beam into a darkened plastic tube fixed at the "finish line." The beam was $2.5 \mathrm{~cm}$ wide and was elevated to a height of $\sim 1 \mathrm{~m}$ above the floor. Each test session consisted of three trials in which latency to cross the beam and the number of foot faults were recorded. 
Rats will normally walk with their feet flat on the surface of the beam (Kolb and Whishaw, 1983). Therefore, a fault was defined as any use of the forelimb or hindlimb on the side of the beam, or if either foot slipped off the top surface of the beam. The results of the three trials were averaged to give a mean latency and foot fault score. Training was completed 1 week before surgery, and testing was done at postsurgical day 15 (before treatment) and again at 4 and 9 weeks after treatment.

\section{Histological procedures}

Histology and volumetric analyses. After the final behavioral test session, animals were given an overdose of sodium pentobarbital and transcardially perfused with $10 \%$ formalin; brains were then harvested. Brains were sectioned at a thickness of 6,40 , or $200 \mu \mathrm{m}$ from a random start point and stained with hematoxylin and eosin, cresyl violet, or GolgiCox (described below), respectively. A uniform random sample of sections between $+3.7 \mathrm{~mm}$ anterior and $-5.8 \mathrm{~mm}$ posterior (relative to bregma) (Paxinos and Watson, 1997) was assessed for infarction, and the area of remaining "good" tissue was measured using the public domain NIH Image program. Infarct areas were determined by projecting a computer-generated grid (Cavalieri Macros; Glen McDonald, University of Washington, Seattle, WA) over a uniformly random sample of sections spanning the extent of the infarct. Based on the Cavalieri principle (Howard and Reed, 1998), unbiased volumes were calculated using the following equation: $V=T \cdot a / p \cdot \Sigma P_{i}$, where $T$ is the thickness of each section, $a / p$ is the known area associated with each grid point, and $P_{i}$ is the number of grid points landing within the infarcted tissue on the $i$ th section. Infarct volumes of the cortex and striatum are represented as percentage of volume of the normal hemisphere. "Infarct" was defined as ischemic tissue that was devoid of neurons (or neuronal processes for Golgi-stained tissue) when examined microscopically.

Golgi-Cox. Animals $(n=24)$ were transcardially perfused with $0.9 \%$ saline, and brains were removed. Whole brains were immersed in a modified Golgi-Cox solution (Glaser and Van der Loos, 1981) for a period of $14 \mathrm{~d}$ before being placed in a $30 \%$ sucrose solution for 2-5 d. Brains were sectioned at $200 \mu \mathrm{m}$ with a vibratome, slide mounted, and stained according to the methods outlined by Gibb and Kolb (1998). Before examination, all slides were coded to conceal the experimental condition.

Both apical and basilar dendrites of layer $\mathrm{V}$ pyramidal cells within the forelimb motor cortex of the uninjured hemisphere were located and drawn at $250 \times$ magnification using the Neurolucida neuron tracing system (MicroBrightField Inc., Colchester, VT). This system allows computer-assisted three-dimensional reconstruction of the dendritic arbor, thus providing accurate measurement of dendrite length. For basilar trees, 10 pyramidal cells were drawn for each animal. However, difficulty in finding intact apical trees (in a few animals) only permitted reconstruction of 6-10 apical dendritic trees per animal. Apical and basilar dendritic trees were examined separately for (1) total dendritic length, (2) total number of branch segments, (3) centrifugal branch ordering (Coleman and Riesen, 1968), and (4) Sholl concentric sphere analysis. Branch order analysis was done such that branches emerging from either the cell body (basilar) or the primary apical dendrite (apical) were first order. After the first bifurcation, branches were considered second order, etc. Quantification of each branch type using this method provides an indication of dendritic arbor complexity.

To be included in the study, cell bodies of pyramidal neurons had to be located within the caudal forelimb region of the motor cortex (extending approximately from bregma to $+2.0 \mathrm{~mm}$ from bregma) as defined by previous anatomical (Zilles and Wree, 1995) and electrophysiological (Neafsey et al., 1986) studies of this region. Also, neurons had to meet a specific selection criterion (Kolb and Gibb, 1991) to be included in the analyses. Briefly, cells had to be completely impregnated, visible in the plane of section, and free from obstruction by other dendrites, astrocytes, or blood vessels. A representative photomicrograph of Golgi-impregnated cells is shown in Figure 2.

\section{Statistics}

All behavioral data were analyzed using a repeated-measures ANOVA or two-way ANOVA when appropriate. Sham groups (SE versus SS) did not significantly differ on behavioral measures; consequently, these groups were pooled for statistical purposes. Infarct volume data were analyzed using unpaired Student's $t$ tests. The dendritic arbor was examined by averaging all cells per animal and subjecting the mean values (for each animal) to ANOVA to determine group differences. Statistical significance for behavioral and anatomical data was ascertained using Newman-Keuls post hoc comparisons.

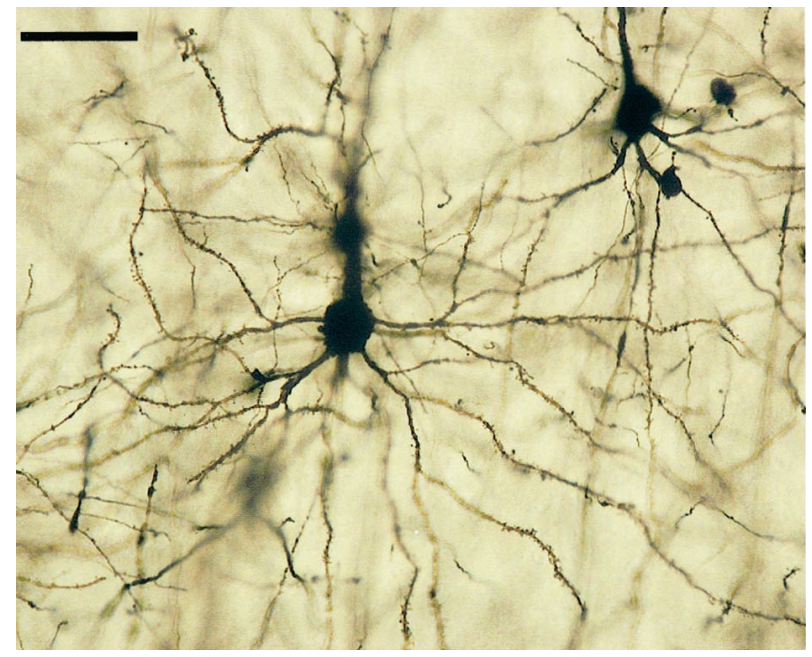

Figure 2. Golgi-Cox-stained cortical tissue. Photomicrograph at $200 \times$ magnification of a layer $\mathrm{V}$ pyramidal cell from the motor cortex demonstrating complete impregnation of dendrites. Scale bar, $100 \mu \mathrm{m}$.

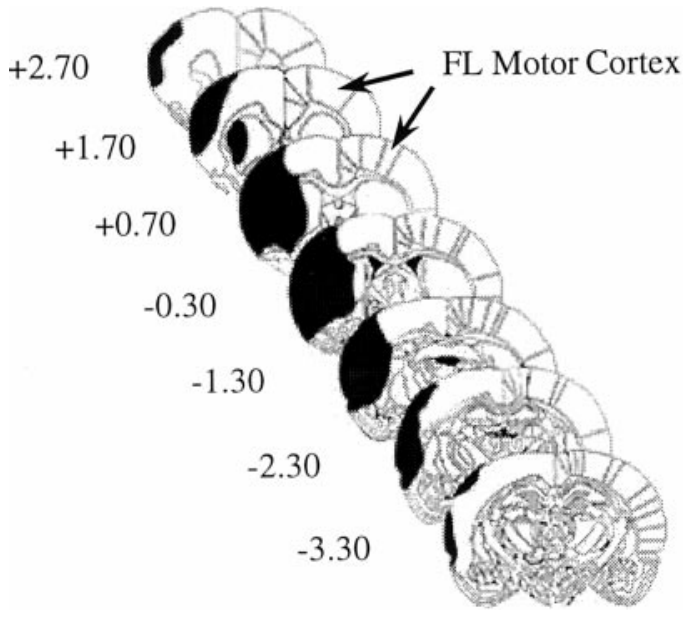

Figure 3. Representative diagram illustrating a typical infarct after ET1-induced MCA occlusion. Note that affected areas include the sensorimotor cortex and the lateral portion of the caudate putamen. Arrows indicate the contralateral forelimb $(F L)$ motor cortex, from which layer $\mathrm{V}$ pyramidal cells were sampled. Stereotaxic coordinates are according to Paxinos and Watson (1997).

\section{RESULTS}

After surgery, all animals were examined for successful MCA occlusion and forelimb impairment. All animals showed vigorous contralateral forelimb retraction-adduction, as well as spontaneous rotation and twisting in the direction contralateral to the lesioned hemisphere when held by the tail.

\section{Infarct volumes}

To assess any possible effect of enriched rehabilitation on infarct size, the volume of the remaining noninfarcted tissue was measured. Figure 3 represents the topographic location of the ischemic infarct, which typically included lateral regions of cortex and lateral striatum (caudate putamen). Infarct size was determined by measuring areas of noninfarcted tissue in both the damaged and undamaged hemispheres. These area measures were then used to calculate values corresponding to the volume of remaining hemispheric, cortical, and striatal tissue. Mean volumetric 
Table 1. Percentage of volume of remaining tissue compared with the contralateral hemisphere

\begin{tabular}{lll} 
& IE & IS \\
\hline Hemisphere & $72.2 \pm 9.2$ & $71.8 \pm 16.3$ \\
Cortex & $59.9 \pm 19.0$ & $61.1 \pm 21.0$ \\
Striatum & $35.45 \pm 25.2$ & $28.9 \pm 20.7$
\end{tabular}

Ischemic infarct size did not statistically differ between the IE and IS groups. Data are mean $\pm \mathrm{SD}$.
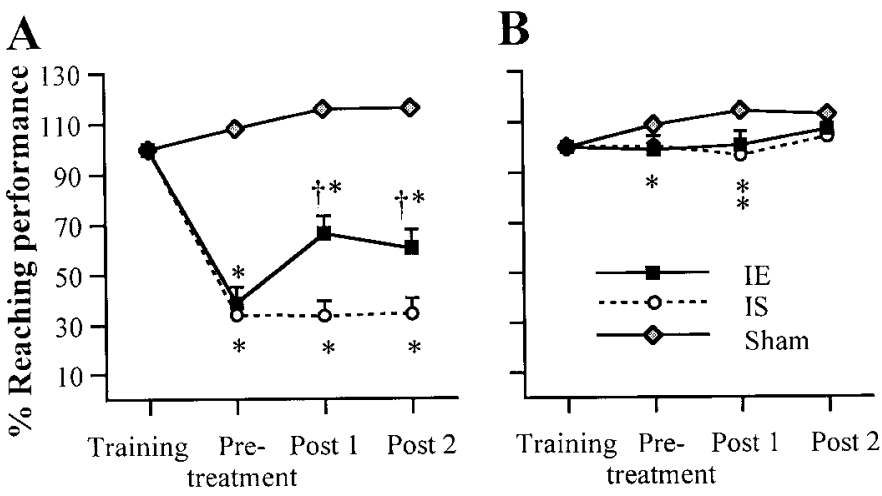

\section{Test Session}

Figure 4. Staircase test of skilled forelimb reaching ability. The percentage for each performance was determined by comparing an individual animal's presurgery training score with subsequent test scores. $A$, Reaching scores for the limb contralateral to the lesion are shown, indicating a sharp impairment after surgery ( $p<0.01$ compared with shams). After 4 weeks (Post 1) of exposure to their respective treatment conditions, IE animals showed a dramatic improvement compared with IS animals $(p<$ 0.01 ), which persisted at 9 weeks after treatment (Post 2). B, Reaching scores for the ipsilateral forelimb show a slight but significant impairment at $10-15 \mathrm{~d}$ after ischemia (before treatment) and at 4 weeks after treatment in both IE and IS animals. Ipsilateral forelimb impairment had recovered to sham levels by 9 weeks of either treatment. Sham groups (SE and SS) were not significantly different at any time point and therefore were pooled for statistical analyses. Data are presented as mean \pm SEM. $*$ indicates different from sham; $\dagger$ indicates different from IS.

values are presented in Table 1. Pathohistological assessment revealed no difference in lesion size between IE and IS animals on either measure (hemisphere, $t_{(1,23)}=0.081, p=0.94$; cortex, $t_{(1,23)}=-0.16, p=0.88$; striatum, $\left.t_{(1,23)}=0.706, p=0.49\right)$.

\section{Skilled reaching}

Figure 4 represents contralateral and ipsilateral reaching performances (respectively) over the course of the experiment. Percentage of reaching scores were obtained by comparing an animal's training performance with its performance on subsequent test sessions. A repeated-measures ANOVA revealed a significant main effect for treatment condition $(F=79.94 ; p<0.0001)$ and day $(F=17.14 ; p<0.001)$, as well as a treatment condition by day interaction effect $(F=9.80 ; p<0.0001)$. Sham animals exposed to either enrichment or standard treatment conditions did not differ in reaching performance for either limb over the duration of the experiment $(p>0.50)$, so these animals were pooled into a single sham group for analysis. At 10-15 d after injury, before assignment to treatment conditions, ANOVA analysis of reaching performance revealed a dramatic impairment in forelimb use contralateral to the lesion $\left(F_{(2,56)}=86.19 ; p<0.0001\right)$ in both ischemic groups compared with shams. Mean reaching performance was not statistically different between IE or IS groups before animals were designated to either treatment condition (38.5 and $33.8 \%$, respectively; $p>0.10$ ). Interestingly, performance with the limb ipsilateral to the infarct also showed a slight but significant decrement in ischemic animals compared with controls at days $10-15\left(F_{(2,56)}=3.64 ; p<0.05\right)$ and at 4 weeks after treatment $\left(F_{(2,56)}=4.48 ; p<0.02\right)$. Ipsilateral reaching in both IE and IS animals remained consistent with training performance but failed to show the same (practice-induced) improvement observed in sham animals (Fig. 4B).

Animals were reassessed for skilled reaching at 4 and 9 weeks after treatment, and a highly significant effect of treatment was observed at both time points (day $30, F_{(2,56)}=66.96, p<0.0001$; day $\left.67, F_{(2,56)}=59.19, p<0.0001\right)$. Post hoc analyses verified that ischemic animals exposed to rehabilitative enrichment performed significantly better than ischemic animals exposed to standard housing $(p<0.01)$. IE animals had improved ( $~ 30 \%$ of pretreatment ability) from the pretreatment test session, whereas the performance of IS animals showed little to no recuperation from early postischemia testing. In addition, tongue use (which is frequently used to retrieve pellets from the top stairs after injury) was almost completely abolished in IE animals demonstrating recovered forelimb use. The observed impairment in forelimb reaching was not attentional because animals persistently (but unsuccessfully) attempted to retrieve food pellets with the impaired limb. Interestingly, the enduring reaching deficit in IE animals appeared to be sensory in nature. After advancing the limb and contacting the pellets, animals seemed unaware that the clasped paw did not contain a pellet because an empty paw was frequently placed into the mouth.

\section{Forelimb asymmetry}

The repeated-measures ANOVA revealed a significant effect of condition for limb use during movements of postural support $(F=16.65 ; p<0.0001)$. Individual ANOVA analysis for each test day revealed a significant group effect at $15 \mathrm{~d}$ after surgery $\left(F_{(2,35)}=20.29 ; p<0.0001\right)$ and at both 4 weeks $\left(F_{(2,35)}=7.66\right.$; $p<0.01)$ and 9 weeks $\left(F_{(2,56)}=18.07 ; p<0.0001\right)$ after treatment. Ischemic groups (IE and IS) showed a significant reliance on the ipsilateral good forelimb for postural support against the cylinder wall at $15 \mathrm{~d}$ after surgery $(p<0.01)$ (Fig. $5 A$ ) compared with sham animals. Interestingly, this preference remained despite being exposed to 4 or even 9 weeks of enriched rehabilitation $(p<0.01)$. Post hoc analyses revealed that IE animals did not differ from IS animals $(p>0.05)$ at pretreatment or after 4 weeks of treatment. At 9 weeks after treatment, IS animals were statistically more dependent on the ipsilateral limb compared with the IE group $(p<0.01)$; however, this difference is likely attributed to the inherent variability of this task. IE animals were not different from earlier test periods (i.e., pretreatment or Post 1 ), which is likely attributable to chance rather than to an improvement of IE animals.

\section{Beam-traversing task}

The beneficial effect observed during skilled reaching was also reflected on a beam-walking test, requiring skilled limb-paw placement to cross the narrow runway. A repeated-measures ANOVA revealed a significant effect of treatment $(F=4.71 ; p<$ $0.05)$ on both total number of foot faults and latency to cross the beam (Fig. $5 B, C$ ). Ischemia (IE and IS) resulted in significantly more foot faults $(p<0.05)$. Remarkably, IE animals displayed marked recovery of limb-placing ability, despite showing substantially greater initial deficits after ischemic injury (before treat- 
Figure 5. A, Asymmetrical forelimb use. Preferential forelimb use was examined at $15 \mathrm{~d}$ after surgery (before treatment) and after 4 and 9 weeks (Post 1 and Post 2, respectively) of exposure to either enrichedrehabilitation or standard-housing conditions. Sham animals did not display a forelimb preference on any test day; however, both IE and IS groups showed a persistent dependence on the ipsilateral good limb $15 \mathrm{~d}$ after ischemia and after 4 and 9 weeks of either treatment $(p<0.01)$. At 9 weeks, the IE group showed significantly less reliance on the ipsilateral forelimb than did the IS group $(p<0.05)$. Sham groups (SE and SS) were not significantly different at any time point; therefore, these groups were pooled for statistical analyses. Black squares, IE; white circles,

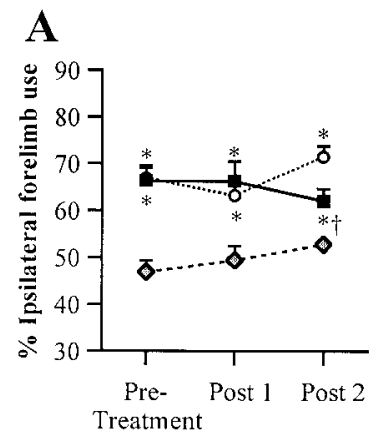

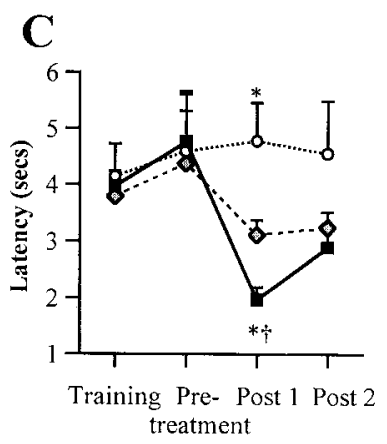

IS; gray diamonds, sham (SE and SS). B, Beam-walking test. After ischemic injury, both the IE and IS groups had a significantly greater number of foot faults compared with sham animals. IS animals showed no spontaneous improvement 4 or 9 weeks later, whereas IE animals were indistinguishable from sham animals after 4 and 9 weeks of enriched rehabilitation. $C$, Mean latency to traverse the beam also indicated improvement in IE animals compared with IS animals. Both the IE and sham groups demonstrated a successive decrease in latency that seemed to correspond with fewer foot faults. IS animals exhibited persistently longer latencies with no apparent improvement. Data are mean \pm SEM. * indicates different from sham; $\dagger$ indicates different from IS.

ment). IE animals were indistinguishable from sham animals after 4 or 9 weeks of treatment, whereas IS animals made significantly more foot faults at all test days $(p<0.05)$.

Latency to traverse the beam was also examined (Fig. $5 C$ ). Group latencies did not differ during training $\left(F_{(2,17)}=0.18 ; p>\right.$ $0.80)$ or on day 15 after surgery $\left(F_{(2,17)}=0.047 ; p>0.90\right)$. At 4 weeks after treatment, latencies decreased significantly for both the sham and IE groups, whereas IS animals were virtually unchanged $\left(F_{(2,17)}=12.91 ; p<0.001\right)$. At 9 weeks after treatment, the trend for longer latencies persisted in IS animals but did not reach statistical significance $\left(F_{(2,17)}=2.65 ; p=0.10\right)$. At Post 1 , IE animals are significantly faster than sham animals $(p<$ $0.05)$. The reason for this is that animals housed in the enriched living condition (both IE and SE animals) traversed the beam more rapidly than did either standard-housed group. Post hoc comparison of IE and SE animals at Post 1 and Post 2 showed that these groups were not significantly different. However, when $\mathrm{SE}$ is pooled with the slower SS animals, the difference becomes statistically significant.

\section{Anatomical analyses}

The dendritic arbor of layer $\mathrm{V}$ pyramidal cells within the undamaged forelimb motor cortex was examined after 9 weeks of either treatment condition and revealed effects for both ischemic lesion and treatment condition. Figure 6 shows representative recon- structions of layer V pyramidal cells from IE, IS, and SE treatment conditions. Importantly, the mean number of basilar branch segments for sham (SS) animals in this study was consistent with previous reports of retrogradely labeled layer $\mathrm{V}$ corticospinal cells (i.e., 40 vs 37 branches, respectively) (Prusky and Whishaw, 1996). Both apical and basilar dendrites were examined; however, no statistical differences in apical branch patterns were observed for any group when measured for dendritic length $\left(F_{(3,19)}=0.56\right.$; $p=0.64)$ or total number of apical dendrite segments $\left(F_{(3,19)}=\right.$ $0.38 ; p=0.78)$.

In contrast, a marked elevation in basilar dendrite arborization was observed. Figure 7, $A$ and $B$, shows the results of basilar dendritic length and the total number of branch segments. ANOVA revealed a significant group effect for total dendritic length per neuron $\left(F_{(3,20)}=7.30 ; p<0.01\right)$. Post hoc analyses confirmed that the IE group had significantly greater dendritic length compared with the IS, SE, and SS groups $(p<0.05)$. A similar pattern was observed for total number of dendritic branch segments per neuron $\left(F_{(3,20)}=7.22 ; p<0.01\right)$ such that IE animals had a significantly greater number of basilar dendrites than SE and SS groups (Fig. 7B). In addition, ischemic injury alone (IS) significantly increased the number of basilar segments compared with SS animals $(p<0.05)$.

The lack of persistent apical alterations was also confirmed by
Figure 6. Representative reconstructions of layer $\mathrm{V}$ pyramidal cells respective of treatment condition (IE, IS, and SE). Note the increased basilar dendritic arbor in the IS group compared with the SE group and even greater complexity in the IE condition. Examination of the apical dendritic tree did not show any statistical differences. Scale bar, $100 \mu \mathrm{m}$.
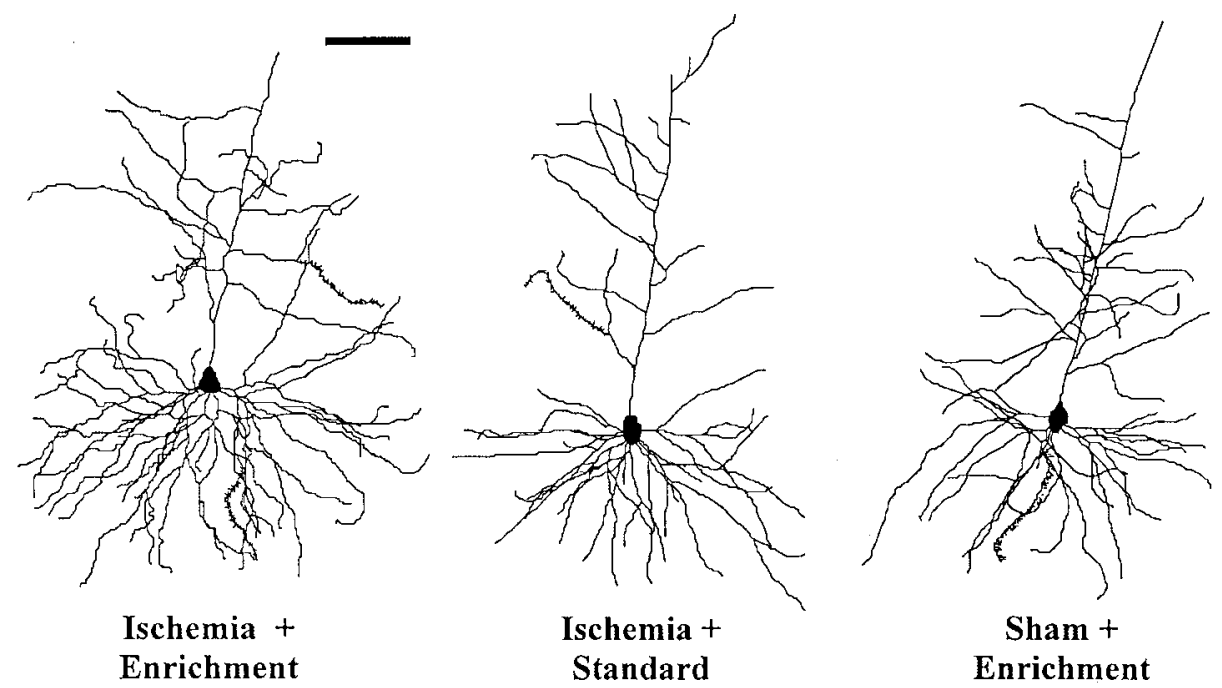

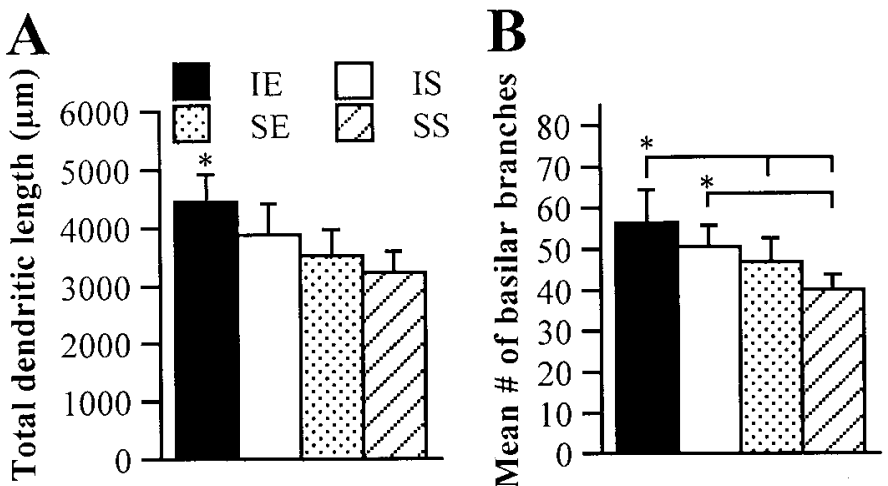

Figure 7. A, Total basilar dendrite length. Enriched rehabilitation (IE) significantly elevated dendritic length compared with the standard-treated ischemic group (IS) and either sham condition (SE and SS). A consistent but nonsignificant increase was observed in the IS condition. $B$, Total number of basilar branch segments per neuron in the undamaged forelimb motor cortex. Enriched rehabilitation resulted in a significantly greater number of branches than was observed in the sham groups (SE or SS). Ischemia alone (IS) also increased branch number compared with SS. Data are mean $\pm \mathrm{SD} .{ }^{*} p<0.05$.

the absence of any group effect for branch number per apical branch order (Fig. $8 A$ ): first, $F_{(3,19)}=1.81, p=0.18$; second, $F_{(3,19)}=1.49, p=0.25$; third, $F_{(3,19)}=0.89, p=0.46$; fourth, $F_{(3,19)}=2.30, p=0.11$; and fifth, $F_{(3,19)}=0.75, p=0.54$. However, elevated basilar arbor complexity was highlighted by a greater number of branch segments per branch order (Fig. $8 B$ ). A significant effect of group was observed at second-order $\left(F_{(3,20)}=\right.$ 3.93; $p<0.05)$, third-order $\left(F_{(3,20)}=5.70 ; p<0.01\right)$, fourth-order $\left(F_{(3,20)}=4.88 ; p<0.01\right)$, fifth-order $\left(F_{(3,20)}=8.36 ; p<0.001\right)$, and sixth-order $\left(F_{(3,20)}=4.95 ; p<0.01\right)$ branches. IE animals had more third-order branches compared with SS animals $(p<$ 0.05 ) and more branches at the fourth, fifth, and sixth order compared with all other groups (IS, SE, and SS groups; $p<0.05$ ).

Figure $8 C$ shows the results of the Sholl concentric sphere analysis, which examines the number of dendrite-sphere intersections at increasing distances from the cell body. IE animals had a consistently greater number of intersections between 60 and $220 \mu \mathrm{m}$ away from the cell body compared with standardhoused sham animals (SS). Significant elevations were observed at $80,100,120,140$, and $160 \mu \mathrm{m}$ from the cell body $(p<0.05)$. Interestingly, IS and SE animals also had an increased number of intersections compared with SS animals but only at distances of 80 and $100 \mu \mathrm{m}(p<0.05)$.

\section{DISCUSSION}

There are several important findings from this study. (1) Enriched rehabilitation after MCA occlusion resulted in a dramatic improvement in skilled use of the impaired forelimb as measured by the reaching and beam-walking tasks. (2) Behavioral recovery was long term ( $\sim 3$ months) and occurred despite treatment being delayed $15 \mathrm{~d}$ after injury and despite ischemic injury to both the cortex and striatum. (3) Ischemic animals exposed to enriched rehabilitation (IE) had significantly greater dendritic arbors in the undamaged contralateral motor cortex compared with all other groups (IS, SE, and SS groups) and were associated with improved functional outcome.

\section{Enriched rehabilitation improves behavioral function}

Previous experiments have demonstrated the functional benefits of exposure to enriched living conditions (Ohlsson and Johans- son, 1995; Johansson and Ohlsson, 1996) or rehabilitative training (Nudo et al., 1996) after focal ischemic injury when either treatment was implemented individually. We hypothesized that, by combining environmental enrichment with task-specific physical rehabilitation, the two conditions would complement each other and further augment neuronal plasticity and recovery. Postinjury enrichment alone does not improve skilled reaching after MCA occlusion (Grabowski et al., 1993). However, our results demonstrate that enriched housing paired with daily reach training improved both skilled reaching and limb placement in beam walking. Similar to other studies (Ohlsson and Johansson, 1995; Johansson and Ohlsson, 1996), enriched rehabilitation did not influence infarct size.

ET-1-induced MCA occlusion caused ischemic injury to the frontoparietal cortex and lateral striatum, resulting in contralateral impairments of forelimb and hindlimb movement and ultimately causing a reliance on the ipsilateral forelimb (Castro, 1977; Whishaw et al., 1986). Exposure to daily enriched rehabilitation resulted in a marked behavioral improvement on both reaching and limb-placement tasks when animals were retested after 4 and 9 weeks of treatment. Standardhoused (IS) animals did not improve. Whishaw (2000) has consistently demonstrated that, after motor cortex lesion, equivalent (compensatory) improvement occurs in skilled reaching, regardless of whether animals receive postsurgical reach training. Such "spontaneous recovery" was not observed in our study, indicating that the severe and persistent impairment ( $\sim 3$ months after stroke) in skilled forelimb use is likely attributable to the compounding effect of both cortical and striatal injury after MCA occlusion. Indeed, the dorsolateral striatum contributes to both initiation and execution of skilled reaching (Whishaw et al., 1986; Pisa, 1988).

Reliance on the unaffected forelimb for upright weightbearing movements persisted in IE animals, despite improved motor function on the reaching and beam-walking tests. This may be attributed to differences in the nature of each test. Forelimb asymmetry examines inherent use of the limb for postural support. In contrast, the staircase test is a "learned" task; animals become skilled in retrieving pellets by precise forelimb movement and digit manipulation. The repeated reaching exercise provided by enriched rehabilitation provides task specificity that improves performance once the animals are retested on the staircase task. Although variations in forelimb movement or posture were not examined directly, motor recovery is likely a result of adaptive compensatory strategies (Whishaw et al., 1991) and is not necessarily comprised of the original posture or forelimb movement patterns. In addition, a process of suppressed movement or "learned nonuse" (Taub et al., 1993) may account for part of the reaching deficits and preferential limb use during postural support. Enriched rehabilitation provided positive reinforcement (i.e., food reward) associated with using the impaired limb, thereby encouraging animals to use "spared" motor function or promoting development of compensatory motor strategies, resulting in lessened functional deficiency.

\section{Effects of enriched rehabilitation on dendritic plasticity}

Increasing evidence from clinical studies of "recovered" stroke patients suggests functional reorganization within peri-infarct regions, as well as the homotopic intact motor cortex (Cramer et al., 1997; Cuadrado et al., 1999; Kopp et al., 1999). Electrolytic lesions of the sensorimotor cortex in rats triggers an 
Figure 8. Branch order and Sholl analyses. $A$, Apical dendritic branch order did not differ among any groups. $B$, Basilar branch order analysis revealed that IE animals had significantly greater numbers of higher-order branches (i.e., 3rd, 4th, 5th, and 6th-plus). IE had significantly more fifth-order and sixth-order or higher branches compared with all other conditions. Interestingly, IS and SE resulted in elevated third-order branches. $C$, Basilar Sholl sphere analysis revealed that IE animals had consistently increased the number of branches between 80 and $260 \mu \mathrm{m}$ away from the cell body. Dendritic

A

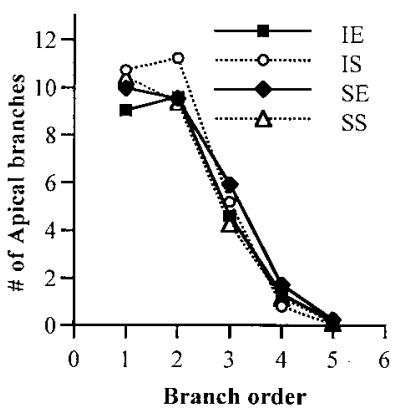

B

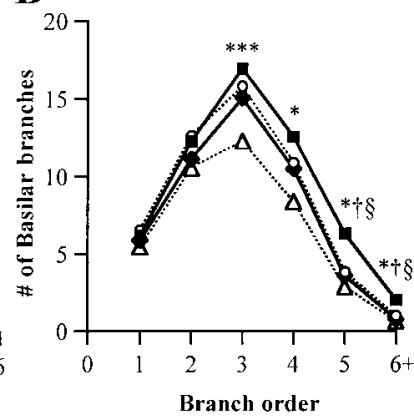

C

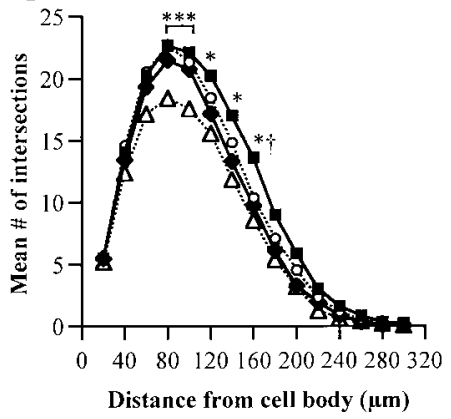

branching in the IE condition was significantly elevated between 80 and $160 \mu \mathrm{m}$. IS and SE conditions were elevated only at 80 and $100 \mu \mathrm{m}$ distances. Error bars have been omitted for clarity. ${ }^{*} p<0.05$ versus SS; $\dagger p<0.05$ versus IS; $\$ p<0.05$ versus SE.

increase in dendritic complexity within the opposite, undamaged sensorimotor cortex (Jones and Schallert, 1992, 1994). Interestingly, if movement of the good limb was restricted, dendritic growth was prevented, and behavioral outcome worsened. Recently, Jones et al. (1999) reported that usedependent synaptogenesis observed in the undamaged hemisphere is enhanced by "acrobat training," suggesting that brain plasticity can be heightened by postinjury rehabilitative measures.

We investigated the effect of deficit-directed rehabilitation on dendritic reorganization (within the intact motor cortex), which may contribute to an enhanced behavioral outcome after focal ischemia in rats. Dendritic growth was only slightly increased in SE animals compared with SS animals. This might be expected because we only measured the untrained hemisphere; therefore, the effect of daily reach training (in shams) is likely restricted to the hemisphere contralateral to the trained limb (Greenough et al., 1985). Interestingly, this suggests that enriched housing did not significantly modify layer $\mathrm{V}$ dendrites.

Ischemic animals exposed to daily enriched rehabilitation showed a pronounced increase in basilar dendritic growth. Alterations in basilar dendrites included total dendritic length, mean number of branch segments, and dendritic arbor complexity. Branch order analysis revealed that the change in dendritic complexity occurred at higher-order branches (i.e., third-order to sixth-order branches), which is indicative of dendritic growth onto existing branches (Kolb et al., 1998). These results are in agreement with those of Jones et al. (1999) such that daily enriched rehabilitation of the impaired forelimb augmented layer $\mathrm{V}$ dendritic plasticity after ischemic injury to the cortex and striatum. This amplified dendritic growth (and probable spine modifications) is likely the substrate for the increased synapse number in layer $\mathrm{V}$ after acrobat training, as reported by Jones et al. (1999). Interestingly, there was an absence of apical dendritic change. One explanation is that apical modifications (as well as any enrichment-induced dendritic changes in sham animals) occurred earlier in the recovery period and were subsequently "pruned" (Jones and Schallert, 1992); however, this possibility could not be determined from this experiment. These dendritic alterations may reflect an ipsilateral component to functional improvement after sensorimotor injury, either by formation of new synaptic connections or alternatively by unmasking redundant pathways (Lee and van Donkelaar, 1995). Indeed, a ventral uncrossed corticospinal tract has been shown in the rat (Vahlsing and Feringa, 1980); however, its role in recovery is unclear. After unilateral cortical lesion, contralateral corticostriatal axons send com- pensatory projections to the (denervated) striatum (Cheng et al., 1998), subsequently causing striatal spine densities to rebound to normal levels, a phenomenon that can be prevented by a second lesion of the contralateral cortex (Cheng et al., 1997). One could speculate that enriched-rehabilitation therapy acts to modify and strengthen the connections that are inherently formed after brain injury, resulting in improved functional outcome.

Ischemic injury produced a modest increase in dendritic arbors in the intact motor cortex that was enhanced by rehabilitative therapy. From this experiment, the physiological mechanism responsible for stimulating this growth is unclear. Motor-skill training, as opposed to motor activity, promotes motor learning associated with dendritic (Greenough et al., 1985) and synaptic (Kleim et al., 1996) modification. Enriched rehabilitation likely enhances learning-induced structural plasticity, which is reflected by improved functional outcome. Training-induced plasticity within motor regions adjacent to the infarct may subsequently affect functionally connected motor circuitry within the intact hemisphere, resulting in dendritic growth. An important consideration is that the observed dendritic changes may be a result of the increased use of the unaffected limb (Jones and Schallert, 1994) in the enriched-rehabilitation condition. Thus, one interpretation is that asymmetrical forelimb use, resulting primarily from striatal injury, produces similar contralateral cortical plasticity as a sensorimotor cortex lesion.

Although our investigation was limited to the contralateral intact motor cortex, numerous studies have provided evidence for extensive neuronal and synaptic remodeling of cortical regions surrounding the lesion (Kolb and Gibb, 1991; Stroemer et al., 1995) and expansion of cortical maps after rehabilitative therapy (Nudo et al., 1996). In the absence of direct anatomical evidence from animal studies to suggest an ipsilateral component to motor recovery, it cannot be concluded that the ipsilateral dendritic modifications reported here are responsible for the observed motor improvement. However, in light of clinical studies (Cramer et al., 1997; Cuadrado et al., 1999; Kopp et al., 1999) that have indicated an ipsilateral contribution to motor recovery, the relevance of ipsilateral morphological changes may be of great importance. Presumably, enriched rehabilitation (even when delayed) augments neuroplastic processes and compensatory neuronal growth within both periinfarct and contralateral tissues, which ultimately contributes to improved motor function.

Intrinsic changes in neurotrophic growth factors resulting from environmental enrichment may also influence neuronal plasticity. Environmental enrichment after focal ischemia alters NGF 
(Dahlqvist et al., 1999) and brain-derived neurotrophic factor (Zhao et al., 2000). In addition, enrichment elevates bFGF in normal animals (Kolb et al., 1998), and after injury bFGF promotes functional recovery and enhanced neuronal growth in both adjacent (Rowntree and Kolb, 1997) and contralateral (Kawamata et al., 1997) brain regions.

In summary, exposure to enriched living conditions in combination with daily forelimb rehabilitation after MCA occlusion greatly enhanced the function of the impaired forelimb. Although compensatory movements may underlie the improved performance, the fact remains that exposure to the enriched physical therapy resulted in greater functionality (i.e., the ability to retrieve more pellets and reduced impairment in limb placement) compared with animals exposed to standard conditions. Importantly, this recovery was long term. Our results suggest that the injured adult rat brain remains sensitive to specific behavioral therapy weeks after the injury. Enriched rehabilitation is capable of augmenting brain plasticity, which was reflected by significantly increased dendritic arbor complexity within the undamaged motor cortex.

Future studies need to establish whether physical rehabilitation initiated at various times throughout the recovery period has differential effects on brain plasticity. For example, early overuse (1-7 d after injury) of the impaired limb caused exacerbation of the lesion and worsened behavioral outcome (Kozlowski et al., 1996). Future studies should consider the type (i.e., electrolytic, aspiration, and ischemia) (Carmichael and Chesselet, 2000; Neumann-Haefelin and Witte, 2000) and location (i.e., cortex versus striatum) of brain injury, as well as the method of promoting limb use (i.e., restrictive cast versus skill training) when investigating postinjury plasticity or the therapeutic efficacy of rehabilitative paradigms. Finally, the present findings provide a significant impetus for more intensive and task-specific rehabilitation of stroke patients.

\section{REFERENCES}

Carmichael ST, Chesselet M-F (2000) Differential gene expression associated with axonal sprouting after focal cortical lesions. Soc Neurosci Abstr 26:114.3.

Castro AJ (1977) Limb preference after lesions of the cerebral hemisphere in adult and neonatal rats. Physiol Behav 18:605-608.

Cheng H-W, Rafols JA, Goshgarian HG, Anavi Y, Tong J, McNeill TH (1997) Differential spine loss and regrowth of striatal neurons following multiple forms of deafferentation: a Golgi study. Exp Neurol 147:287-298.

Cheng H-W, Tong J, McNeill TH (1998) Ultrastructural evidence for cortical axon sprouting in the deafferented striatum of adult rat. Neurosci Lett 241:1-4.

Coleman PD, Riesen AH (1968) Environmental effects on cortical dendritic fields. I. Rearing in the dark. J Anat 102:363-374.

Cramer SC, Nelles G, Benson RR, Kaplan JD, Parker RA, Kwong KK, Kennedy DN, Finklestein SP, Rosen BR (1997) A functional MRI study of subjects recovered from hemiparetic stroke. Stroke 28:2518-2527.

Cuadrado ML, Egido JA, Gonzalez-Gutierrez JL, Varela-de-Seijas E (1999) Bihemispheric contribution to motor recovery after stroke: a longitudinal study with transcranial Doppler ultrasonography. Cerebrovasc Dis 9:337-344.

Dahlqvist P, Zhao L, Johansson I-M, Mattsson B, Johansson BB, Seckl JR, Olsson T (1999) Environmental enrichment alters nerve growth factor-induced gene $\mathrm{A}$ and glucocorticoid receptor messenger RNA expression after middle cerebral artery occlusion in rats. Neuroscience 93:527-535.

Forgie ML, Gibb R, Kolb B (1996) Unilateral lesions of the forelimb area of rat motor cortex: lack of evidence for use-dependent neural growth in the undamaged hemisphere. Brain Res 710:249-259.

Gibb R, Kolb B (1998) A method for vibratome sectioning of Golgi-Cox stained whole rat brain. J Neurosci Methods 79:1-4.

Glaser EM, Van der Loos H (1981) Analysis of thick brain sections by obverse-reverse computer microscopy: application of a new, high clarity Golgi-Nissl stain. J Neurosci Methods 4:117-125.
Grabowski M, Brundin P, Johansson BB (1993) Paw-reaching, sensorimotor, and rotational behavior after brain infarction in rats. Stroke 24:889-895.

Greenough WT, Larson JR, Withers GS (1985) Effects of unilateral and bilateral training in a reaching task on dendritic branching of neurons in the rat motor-sensory forelimb cortex. Behav Neural Biol 44:301-314.

Howard CV, Reed MG (1998) Unbiased stereology: three-dimensional measurement in microscopy. New York: Springer.

Johansson BB, Ohlsson AL (1996) Environment, social interaction, and physical activity as determinants of functional outcome after cerebral infarction in the rat. Exp Neurol 139:322-327.

Jones TA, Schallert T (1992) Overgrowth and pruning of dendrites in adult rats recovering from neocortical damage. Brain Res 581:156-160.

Jones TA, Schallert T (1994) Use-dependent growth of pyramidal neurons after neocortical damage. J Neurosci 14:2140-2152.

Jones TA, Chu CJ, Grande LA, Gregory AD (1999) Motor skills training enhances lesion-induced structural plasticity in the motor cortex of adult rats. J Neurosci 19:10153-10163.

Kawamata K, Dietrich WD, Schallert T, Gotts JE, Cocke RR, Benowitz LI, Finklestein SP (1997) Intracisternal basic fibroblast growth factor enhances functional recovery and up-regulates the expression of a molecular marker of neuronal sprouting following cerebral infarction. Proc Natl Acad Sci USA 94:8179-8184.

Kleim JA, Lussnig E, Schwarz ER, Comery TA, Greenough WT (1996) Synaptogenesis and Fos expression in the motor cortex of the adult rat after motor skill learning. J Neurosci 16:4529-4535.

Kolb B, Gibb R (1991) Environmental enrichment and cortical injury: behavioral and anatomical consequences of frontal cortex lesions in rats. Cereb Cortex 1:189-198.

Kolb B, Whishaw IQ (1983) Dissociation of the contributions of the prefrontal, motor, and parietal cortex to the control of movement in the rat: an experimental review. Can J Psychol 37:211-232.

Kolb B, Forgie M, Gibb R, Gorny G, Rowntree S (1998) Age, experience, and the changing brain. Neurosci Biobehav Rev 22:143-159.

Kopp B, Kunkel A, Muhlnickel W, Villringer K, Taub E, Flor H (1999) Plasticity in the motor system related to therapy-induced improvement of movement after stroke. NeuroReport 10:807-810.

Kozlowski DA, James DC, Schallert T (1996) Use-dependent exaggeration of neuronal injury after unilateral sensorimotor cortex lesions. J Neurosci 16:4776-4786.

Kunkel A, Kopp B, Miller G, Villringer K, Villringer A, Taub E, Flor H (1999) Constraint-induced movement therapy for motor recovery in chronic stroke patients. Arch Phys Med Rehabil 80:624-628.

Lee RG, van Donkelaar P (1995) Mechanisms underlying functional recovery following stroke. Can J Neurol Sci 22:257-263.

Liepert J, Bauder H, Miltner WHR, Taub E, Weitller C (2000) Treatment-induced cortical reorganization after stroke in humans. Stroke 31:1210-1216.

Montoya CP, Campbell-Hope LJ, Pemberton KD, Dunnett SB (1991) The "staircase test": a measure of independent forelimb reaching and grasping abilities in rats. J Neurosci Methods 36:219-228.

Neafsey EJ, Bold EL, Haas G, Hurley-Guis KM, Quirk G, Sievert CF, Terreberry RR (1986) The organization of the rat motor cortex: a microstimulation mapping study. Brain Res Rev 11:77-97.

Neumann-Haefelin T, Witte OW (2000) Periinfarct and remote excitability changes after transient middle cerebral artery occlusion. J Cereb Blood Flow Metab 20:45-52.

Nudo RJ, Wise BM, SiFuentes F, Milliken GW (1996) Neural substrates for the effects of rehabilitative training on motor recovery after ischemic infarct. Science 272:1791-1794.

Ohlsson A-L, Johansson BB (1995) Environment influences functional outcome of cerebral infarction in rats. Stroke 26:644-649.

Paxinos G, Watson C (1997) The rat brain in stereotaxic coordinates. San Diego: Academic.

Pisa M (1988) Motor functions of the striatum in the rat: critical role of the lateral region in tongue and forelimb reaching. Neuroscience 24:453-463.

Prusky G, Whishaw IQ (1996) Morphology of identified corticospinal cells in the rat following motor cortex injury: absence of use-dependent change. Brain Res 714:1-8.

Risedal A, Zeng J, Johansson BB (1999) Early training may exacerbate brain damage after focal brain ischemia in the rat. J Cereb Blood Flow Metab 19:997-1003.

Rosenzweig MR, Krech D, Bennett EL, Diamond MC (1962) Effects of environmental complexity and training on brain chemistry and anatomy: a replication and extension. J Comp Physiol Psychol 55:429-437.

Rowntree S, Kolb B (1997) Blockade of basic fibroblast growth factor retards recovery from motor cortex injury in rats. Eur J Neurosci 9:2432-2441.

Sharkey J, Butcher SP (1995) Characterisation of an experimental model of stroke produced by intracerebral microinjection of endothelin-1 adjacent to the rat middle cerebral artery. J Neurosci Methods 60:125-131. 
Sharkey J, Ritchie IM, Kelly PAT (1993) Perivascular microapplication of endothelin-1: a new model of focal ischemia in the rat. J Cereb Blood Flow Metab 13:865-871.

Stroemer RP, Kent TA, Hulsebosch CE (1995) Neocortical neural sprouting, synaptogenesis, and behavioral recovery following neocortical infarction in rats. Stroke 26:2135-2144.

Taub E, Miller NE, Novack TA, Cook EW, Fleming WC, Nepomuceno CS, Connell JS, Crago JE (1993) Technique to improve chronic motor deficit after stroke. Arch Phys Med Rehabil 74:347-354.

Vahlsing HL, Feringa ER (1980) A ventral uncrossed corticospinal tract in the rat. Exp Neurol 70:282-287.

Whishaw IQ (2000) Loss of innate cortical engram for action patterns used in skilled reaching and the development of behavioral compensation following motor cortex lesions in the rat. Neuropharmacology 39:788-805.
Whishaw IQ, O'Connor RB, Dunnett SB (1986) The contributions of the motor cortex, nigrostriatal dopamine, and caudate-putamen to skilled forelimb use in the rat. Brain 109:805-843.

Whishaw IQ, Pellis SM, Gorny BP, Pellis VC (1991) The impairments in reaching and the movements of compensation in rats with motor cortex lesions: an endpoint, videorecording, and movement notation analysis. Behav Brain Res 42:77-91.

Zhao LR, Mattsson B, Johansson BB (2000) Environmental influence on brain-derived neurotrophic factor messenger RNA expression after middle cerebral artery occlusion in spontaneously hypertensive rats. Neuroscience 97:177-184.

Zilles K, Wree A (1995) Cortex: areal and laminar structure. In: The rat nervous system, Ed 2 (Paxinos G, ed), pp 649-685. San Diego: Academic. 